with any STI was 19.5 years. $102(51.0 \%)$ of the women were using different methods of hormonal contraceptives while 84 (42.0\%) had intrauterine contraceptives devices inserted. About $54.5 \%$ (109) had various STIs in both groups. The most common STI diagnosed was bacterial vaginosis $(26.5 \%)$. Other STIs diagnosed were vaginal candidiasis $(26.0 \%)$, HIV (8.5\%), trichomoniasis (7.5\%), Chlamydia cervicitis $(7.0 \%)$, syphilis $(3.5 \%)$, Genital warts $(3.5 \%)$ and gonorrhoea $(2.5 \%)$. Even though bacterial vaginosis and candidiasis were diagnosed more in women with IUCD while Chlamydia cervicitis, syphilis and gonorrhoea occurred more in women using various types of hormonal contraceptives, there was no statistical relationship between the STIs and their sexual behaviours.

Conclusion Women seeking contraception to prevent unintended pregnancy are as much in need of education about prevention of STIs as much as the counselling regarding the most effective contraceptive methods. Younger age, increased numbers of sexual partners and oral mode of sexual intercourse were significantly associated with increased risk of acquiring vaginal tric.

\section{P1-S5.04 HSV-2 PREVALENCE AND INCIDENCE AMONG STABLE HIV-1 DISCORDANT COUPLES IN NAIROBI, KENYA}

\section{doi:10.1136/sextrans-2011-050108.182}

A Muiru, R Bosire, B Guthrie, R Choi, M Merkel, B Chohan, J Kiarie, B Lohman-Payne, C Farquhar. Kenya Research Group, International AIDS Research and Training Program, Seattle, USA

Background To describe HSV-2 prevalence, incidence and their correlates among stable HIV-1 discordant heterosexual couples enrolled in an HIV-1 prevention cohort study in Nairobi, Kenya.

Methods Between 2007 and 2009, 469 HIV-1 discordant couples were recruited from VCT centers, and were followed up quarterly for up to 2 years. Clinical staff administered a questionnaire assessing socio-demographics, behaviour, and biological characteristics. HSV-2 status was assessed using Focus Herpe-Select2 HSV IgG ELISA. Correlates of HSV-2 infection at enrolment, and during follow-up were identified by logistic regression and Cox proportional hazards analysis respectively.

Results Of the couples, 189 (40\%) were HSV-2 concordant positive, 114 (24\%) were HSV-2 discordant, and 85 (18\%) were concordant negative. The incidence of HSV-2 infection was 8.7 cases/100 person-years. $19(17 \%)$ individuals in an initially HSV-2 discordant partnership and $11(13 \%)$ in an initially HSV-2 concordant negative partnership acquired HSV-2 during follow-up. Of these 11 individuals, 9(81\%) were the HIV-1 positive index case in the HIV-1 discordant relationship. In a multivariate analysis, HSV-2 prevalence at baseline was significantly associated with female gender (OR 4.4; (95\% CI 2.9 to 6.7)), having an HSV-2 positive partner (OR 7.6; (95\% CI 5.1 to 11.2$)$ ), being HIV positive (OR 2.2; (95\% CI 1.5 to $3.1)$ ), increasing age (OR 1.08; (95\% CI 1.05 to 1.1$)$ ), and older age at sexual debut was protective (OR 0.87; (95\% CI 0.83 to 0.93 )). HSV-2 incidence during follow-up was significantly associated with female gender (HR 3.5; (95\% CI 1.6 to 8.0)), having an HSV-2 positive partner (HR 4.9; (95\% CI, 2.0 to 9.9)), and history of any other STI (HR 2.6; (95\% CI 1.3 to 5.8)).

Conclusions This is the first large study to report the prevalence and incidence of HSV-2 among sexual partners who are in a stable HIV-1 discordant relationship. Female gender, a history of any other STI, and having an HSV-2 positive partner were associated with an increased risk of acquiring HSV-2. 11 individuals a majority of them with HIV acquired HSV-2 during follow-up despite being in HSV-2 concordant negative relationship at enrolment. The high incidence of HSV-2 in couples who were initially HSV-2 concordant negative or HSV-2 discordant highlights the dire need for continuing comprehensive STD prevention programs particularly among high risk population Sub-Saharan Africa.

\section{P1-S5.05 THE EMERGENCE OF HIV PANDEMIC: WHO LIFTED THE LID OF THE AFRICAN SIV-HIV POT? A HYPOTHESIS}

doi:10.1136/sextrans-2011-050108.183

${ }^{1}$ A Lovenetski. ${ }^{1}$ NEARMEDIC PLUS, Moscow, Russian Federation

A series of studies established that HIV infection in humans originated from multiple episodes of zoonotic transmission of CD4 T cell-tropic lentiviruses infecting African monkey species-Simian Immunodeficiency Viruses (SIV). HIV-1 derives from the chimpanzee (Pan troglodytes)-SIVcpz, while HIV-2 derives from SIVsm, which naturally infects the Sooty mangabey (Cercocebus atys). Pan troglodytes is present across West Central Africa. Cercocebus atys have their natural habitat in West Africa (Guinea-Bissau to Cote d'Ivoire). Timing the emergence of HIV-1 in humans: four of the earliest known instances of HIV-1 infection are as follows: A plasma sample taken in 1959 from an adult male living in the Democratic Republic of the Congo. A lymph node sample taken in 1960 from an adult female, also from the Democratic Republic of the Congo. HIV-1 found in tissue samples from an American teenager who died in St. Louis in 1969. HIV-1 found in tissue samples from a Norwegian sailor who died around 1976. An 1998 analysis of the plasma sample dated 1959 suggested that HIV-1 was introduced into humans around the 1940s or the early 1950s. In 2000 the results of a new study suggested that HIV-1 infection occurred around 1931 in West Africa. However, a study in 2008 dated the origin of HIV to between 1884 and 1924 . Timing the emergence of HIV-2 in human population: in 2003 molecular timing analysis of two subtypes of HIV-2 (A and B) and SIVsm samples led to conclusion that subtype $A$ had passed into humans around 1940 and subtype B in 1945. The data evidence that Africa was the continent where the transfer of emerged HIV-1 and HIV-2 to humans first occurred. What caused the HIV epidemic to spread so suddenly from African pot in late 70s and early 80s? In this connection let's recall the largest successful worldwide medical intervention in human history-the WHO Smallpox Eradication Programme (1967-1977). Smallpox epidemics had inflicted mankind throughout history, and during 1967 some 10-15 million cases were occurring in 30 endemic countries. In WHO monograph Smallpox and Its Eradication "the chronology and precise timing of the Programme progress was well documented including Africa region: at the end of 1971 smallpox was endemic in only three African countries and was completely eradicated by 1977 . Can there be a possible link between the HIV-1/HIV-2 pandemic emergence from West and Central Africa and smallpox eradication?. Smallpox was endemic for most of the countries of West and Central Africa where HIV's originated. Smallpox may be fatal for the most of the HIV-infected immunocompromised persons resulting in interruption of HIV spread. Thus smallpox could be considered as a natural barrier that limited prevalence of newly emerged HIV-1/HIV-2 in local human population. Smallpox eradication probably was the key factor which may contribute the widespread of HIV's in the population of West and Central Africa and the subsequent HIV pandemic emergence".

\section{Epidemiology poster session 5: Transmission dynamic + behaviour}

\section{P1-S5.06 ORAL SEX AND RISK OF SEXUALLY TRANSMITTED INFECTIONS}

doi:10.1136/sextrans-2011-050108.184

${ }^{1} \mathrm{E}$ Flagg, ${ }^{1} \mathrm{H}$ Weinstock, ${ }^{1,2} \mathrm{~K}$ Workowski, ${ }^{1} \mathrm{~T}$ Peterman. ${ }^{1} \mathrm{US}$ Centers for Disease Control and Prevention, Atlanta, USA; ${ }^{2}$ Emory University, USA

Background Oral sex is a common sexual practice in the United States; in 2002, almost $90 \%$ of sexually active adults ages $20-44$ and 
over half of teens 15-19 years reported having had oral sex with an opposite sex partner. Oral sex is thought by some men who have sex with men to have lower risk for HIV acquisition, and is a prevalent practice in this population. However, the risk of acquiring a given sexually transmitted infection (STI) from oral sex may depend on a number of factors, including the specific STI, particular oral sex practice, prevalence of the STI in the population, and the number of oral sex acts performed. It is difficult to quantify the risk of getting an STI from a specific type of oral sex activity, or compare risks due to specific sex acts, because most people who have oral sex also have vaginal and/or anal sex.

Methods In order to develop public health messages on oral sex and STI risk, we performed a review of the scientific literature to assess the state of current knowledge for risks of seven STIs (including HIV) and six oral sex practices: insertive and receptive fellatio, cunnilingus, and anilingus. Approximately 200 publications between 1979 and 2010 were reviewed; only 65 reported novel findings for one or more of these 42 potential associations.

Results The clearest evidence of risk was found for syphilis (all oral sex practices), chlamydia (insertive and receptive fellatio; receptive anilingus), gonorrhoea (insertive and receptive fellatio), herpes (insertive fellatio; receptive cunnilingus), and HIV (receptive fellatio). For example, for pharyngeal gonorrhoea 4 of 4 studies reported association with receptive fellatio; 1 of 1 study found none for insertive anilingus. Other STI and oral sex associations were less well-studied, or have not been documented. No reports allow direct per-contact estimates, but some facilitate comparisons with non-oral types of sexual contact. A number of studies did not specify whether ejaculation or use of barrier protection was assessed.

Conclusions All oral sex practices are associated with risk of transmission of one or more STIs; however the relations between specific oral sex practices and risk of infection are not well-characterised for several STIs. Despite this, it is important to formulate appropriate public health messages regarding oral sex and STI risk. Future studies should clearly distinguish between specific oral sex practices and consider these factors in sample size determination.

\section{P1-S5.07 ONGOING RISK BEHAVIOURS: NEW STD DIAGNOSES IN PERSONS KNOWN TO BE HIV-INFECTED IN MIAMI-FORT LAUDERDALE (SOUTH FLORIDA), 2000-2009}

doi:10.1136/sextrans-2011-050108.185

${ }^{1} \mathrm{~T}$ A Peterman, ${ }^{2} \mathrm{~T}$ H Brewer, ${ }^{1} \mathrm{D}$ R Newman, ${ }^{3} \mathrm{~S}$ Shiver. ${ }^{1} \mathrm{CDC}$, Atlanta, USA; ${ }^{2} \mathrm{CDC} /$ Florida DOH, Miami, USA; ${ }^{3}$ Florida Department of Health, Tallahassee, USA

Background The diagnosis of a new bacterial STD in a person with documented HIV- infection suggests ongoing high-risk sexual behaviours. While high rates of syphilis among HIV-positive MSM have been repeatedly noted in South Florida, little attention has been given to the incidence of gonorrhoea (GC) and chlamydia (CT) among HIV-positive individuals, due to use of separate surveillance systems, differing security requirements, and limited partner services staff available to investigate GC and CT cases.

Methods We reviewed STD surveillance data from 2000 to 2009 after linking it to the HIV/AIDS surveillance data base. We analysed cases of GC and CT diagnosed $>60$ days after an HIV diagnosis.

Results Overall $3.7 \%$ of GC (1504 of 40214 ) and $1.2 \%$ of CT (1149/ 99265) cases were in persons known to be HIV- positive. The percentage of cases diagnosed in HIV- positive persons increased steadily over the time period; GC coinfections increased from $2.3 \%$ in 2000 to $5.0 \%$ in 2009 and CT coinfections increased from $0.8 \%$ to $1.3 \%$. Most of the GC coinfected cases were among males; 1144/
$21360(5.4 \%)$ of males with GC were coinfected with HIV while $360 / 18854(1.9 \%)$ of females with GC were coinfected with HIV. For CT, $559 / 22735(2.5 \%)$ of males with CT were coinfected with HIV while $590 / 76530$ (0.8\%) of females with CT were coinfected with HIV. Most coinfections were among HIV positive males, 73.5\% of whom were men who have sex with men (MSM). Coinfections among men increased over the time period, while among women the number peaked in 2006 and has declined thereafter. HIV-positive men accounted for over $10 \%$ of GC infections among men $35-59$ years of age and over $10 \%$ of CT infections among males 40-59. HIV coinfection was present for over $5 \%$ of GC infections among women $30-44$ years of age and $50-54$ years of age.

Conclusions While males with GC or CT who are coinfected with HIV constitute a small fraction of the cases in South Florida, the proportion is increasing steadily. HIV+ MSM account for a high percentage of cases among males over 35 years of age. Timely, accessible linkage between HIV and STD databases is essential to direct partner services and risk reduction counselling to this high-risk population.

\section{P1-S5.08 ATTENDANCE AT LOCAL AND NATIONAL SEX-EVENTS ASSOCIATED WITH STD DIAGNOSIS, SAN FRANCISCO, 2010}

doi:10.1136/sextrans-2011-050108.186

K Bernstein, H Hjord, J Marcus, R Kohn, S Philip. San Francisco Department of Public Health, San Francisco, USA

Background A variety of sex-themed events draw crowds from across the USA and provide opportunities for sex partner recruitment, potentially amplifying local sexual networks and complicating disease prevention and control. We examined the frequency of attendance at such events and the association between attendance and incident STD diagnosis among patients visiting a municipal STD clinic.

Methods During 21 September 10-1 November 10, patients seeking services at San Francisco City Clinic were invited to complete a survey about their attendance at local and national events in the prior 12 months, including street fairs, gay pride events, Burning Man, leather events, and circuit parties. Survey results were linked with data from the clinic visit, including self-reported risk behaviours and STD diagnoses. Analyses were stratified by sexual orientation. Events traditionally associated with sex partner recruitment (sex events) were combined. $\chi^{2}$ and Wilcoxon rank-sum statistics were used to compare patients who attended sex events with all other patients completing the survey.

Results Of the 246 completed surveys, 106 (43.1\%) were among heterosexual patients and 140 (56.9\%) were among men who have sex with men (MSM). Twenty per cent of heterosexual patients and $47 \%$ of MSM patients reported attending a sex event in the prior 12 months. Among heterosexual patients, those attending a sex event were of younger age (median 25 vs 29 years, $p=0.014)$, reported fewer sex partners in the prior 12 months (median 1 vs $2, p=0.021$ ) and were more likely to be at their first visit to the STD clinic $(71.4 \%$ vs $47.1 \%, 0.045)$. There was no association between STD diagnosis and event attendance among heterosexuals. Among MSM, those attending sex events reported more sex partners in the prior 12 months (median $10 \mathrm{vs} 5, \mathrm{p}=0.019$ ), were more likely to be residents of San Francisco (83.3\% vs $66.2 \%$, $\mathrm{p}=0.021$ ), and were more likely to be diagnosed with chlamydia ( $15.2 \%$ vs $4.1 \%, p=0.024)$ or any STD $(29.0 \%$ vs $14.9 \%, p=0.045)$ at that visit.

Conclusions Attendance at sex events was common among patients at an urban STD clinic. Among MSM, attendance was associated with more sex partners and STD diagnosis. The ability of local 